\title{
Synchrotron experiments revealing the similarities and differences between crystal and enzyme environmental effects on the electron densities of protease inhibitors and ibuprofen derivatives
}

\author{
S. Grabowsky \\ University of Bern, Department of Chemistry, Biochemistry and Pharmaceutical Sciences, Freiestrasse 3, 3012 Bern, Switzerland \\ simon.grabowsky@dcb.unibe.ch
}

Non-covalent intermolecular interactions polarize a drug molecule in the biological environment to prepare it for the recognition and binding process with a related enzyme. In a crystal structure of the same drug molecule, the crystal packing is defined by the same kind of non-covalent interactions. This means that in both a biological as well as a crystalline environment, the small molecule will conformationally adapt its shape to the prevailing intermolecular binding forces, so that the resulting bound state reflects both its inherent flexibility and the environment. Electrostatic complementarity between an enzyme binding site and an active molecule is an aspect that goes beyond geometry and molecular conformation since the electrostatic potential is inherently related to the electron density distribution. We ask to which extent small-molecule crystal structures can be used to predict the conformation and interaction density of the same molecule in the enzyme.

The first compound class investigated is related to loxistatin acid E64c. These compounds are cysteine protease inhibitors, and the active site is an electrophilic epoxide ring.[1] It took us many years to solve the small-molecule crystal structure of E64c,[2] and experimental electron-density studies were only possible for related model compounds.[3] Recently, however, we were able to perform a full quantum-crystallographic, molecular-dynamics and QM/MM study of the active site of E64c co-crystallizing in a system that closely resembles the binding situation of E64c in the cysteine protease cathepsin B.[4]

The second compound class investigated refers to ibuprofen derivatives. We used the umpolung principle to tune the properties of ibuprofen by carbon-silicon exchange, which in turn impacts on the electrostatic complementarity relationships when ibuprofen binds to cyclooxygenases.[5] Again, we investigated the enzyme and crystal environmental effects on ibuprofen and sila-ibuprofen by quantum crystallography, molecular dynamics and QM/MM calculations.[6]

Every low-temperature high-resolution single-crystal X-ray diffraction experiment utilized in this study was conducted at a synchrotron beamline at either DESY, APS or SPring-8. Without access to large infrastructure, such studies on weakly scattering pharmaceutically active compounds would not be possible. I will therefore not only report the biochemically relevant results, but also the importance of synchrotron experiments for our field.

[1] Mladenovic, M., Ansorg, K., Fink, R. F., Thiel, W., Schirmeister, T. \& Engels, B. (2008). J. Phys. Chem. B, $112,11798$.

[2] Shi, M. W., Sobolev, A. N., Schirmeister, T., Engels, B., Schmidt, T. C., Luger, P., Mebs, S., Dittrich, B., Chen, Y.-S., Bąk, J. M., Jayatilaka, D., Bond, C. S., Turner, M. J., Stewart, S. G., Spackman, M. A. \& Grabowsky, S. (2015). New J. Chem. 39, 1628.

[3] Grabowsky, S., Schirmeister, T., Paulmann, C., Pfeuffer, T. \& Luger, P. (2011). J. Org. Chem. 76, 1305.

[4] Kleemiss, F., Wieduwilt, E. K., Hupf, E., Shi, M. W., Stewart, S. G., Jayatilaka, D., Turner, M. J., Sugimoto, K., Nishibori, E., Schirmeister, T., Schmidt, T. C., Engels, B. \& Grabowsky, S. (2021). Chem. Eur. J. 27, 3407.

[5] Kleemiss, F., Justies, A., Duvinage, D., Watermann, P., Ehrke, E., Sugimoto, K., Fugel, M., Malaspina, L. A., Dittmer, A., Kleemiss, T., Puylaert, P., King, N. R., Staubitz, A., Tzschentke, T. M., Dringen, R., Grabowsky, S. \& Beckmann, J. (2020). J. Med. Chem. 63, 12614.

[6] Kleemiss, F., Duvinage, D., Puylaert, P., Fugel, M., Sugimoto, K., Beckmann, J. \& Grabowsky, S. (2021). Acta Cryst. B, in preparation.

Keywords: synchrotron X-ray diffraction, experimental charge density, quantum crystallography, environmental effects, drug molecules 